OPEN ACCESS

Edited by:

Timur Ekiz,

Turkmenbasi Medical Center, Turkey

Reviewed by:

Kamal Mezian,

Charles University, Czechia

Bayram Kelle,

Cukurova University, Turkey

*Correspondence:

Chueh-Hung Wu

b88401062@ntu.edu.tw

Specialty section:

This article was submitted to

Neuropharmacology,

a section of the journal

Frontiers in Pharmacology

Received: 04 November 2020 Accepted: 26 November 2020

Published: 17 December 2020

Citation:

Lin M-T, Liao C-L, Hsiao M-Y, Hsueh H-W, Chao C-C and Wu C-H (2020) Volume Matters in Ultrasound-

Guided Perineural Dextrose Injection

for Carpal Tunnel Syndrome: A Randomized, Double-Blinded, Three-

Arm Trial.

Front. Pharmacol. 11:625830

doi: $10.3389 /$ fphar.2020.625830

\section{Volume Matters in Ultrasound-Guided Perineural Dextrose Injection for Carpal Tunnel Syndrome: A Randomized, Double-Blinded, Three-Arm Trial}

\author{
Meng-Ting Lin ${ }^{1}$, Chun-Li Liao ${ }^{1}$, Ming-Yen Hsiao ${ }^{2}$, Hsueh-Wen Hsueh ${ }^{3}$, Chi-Chao Chao ${ }^{3}$ and \\ Chueh-Hung $\mathrm{Wu}^{2,4 *}$
}

${ }^{1}$ Department of Physical Medicine and Rehabilitation, National Taiwan University Hospital Hsin-Chu Branch, Hsinchu, Taiwan, ${ }^{2}$ Department of Physical Medicine and Rehabilitation, National Taiwan University Hospital, College of Medicine, National Taiwan University, Taipei, Taiwan, ${ }^{3}$ Department of Neurology, National Taiwan University Hospital, Taipei, Taiwan, ${ }^{4}$ Department of General Medicine, National Taiwan University Hospital Biomedical Park Branch, Hsinchu, Taiwan

Ultrasound-guided perineural dextrose injection (PDI) has been reported effective for carpal tunnel syndrome (CTS). Higher volume of injectate may reduce adhesion of median nerve from other tissues, but volume-dependent effects of PDI in CTS remain unknown. We aimed to investigate whether PDI with different injectate volumes had different effects for CTS participants. In this randomized, double-blinded, three-arm trial, 63 wrists diagnosed with CTS were randomized into three groups that received ultrasound-guided PDI with either 1, 2 or $4 \mathrm{ml}$ of $5 \%$ dextrose water. All participants finished this study. Primary outcome as visual analog scale (VAS) and secondary outcomes including Boston Carpal Tunnel Questionnaire (BCTQ), Disability of the Arm, Shoulder and Hand score (QuickDASH), electrophysiological studies and cross-sectional area (CSA) of the median nerve at carpal tunnel inlet were assessed before and after PDI at the 1st, 4th, 12th and 24th weeks. For within-group analysis, all three groups (21 participants, each) revealed significant improvement from baseline in VAS, BCTQ and QuickDASH at the 1st, 4th, 12th and 24th weeks. For between-group analysis, $4 \mathrm{ml}$-group yielded better VAS reduction at the 4th and 12th weeks as well as improvement of BCTQ and QuickDASH at the 1st, 4th, and 12th weeks, compared to other groups. No significant between-group differences were observed in electrophysiological studies or median nerve CSA at any follow-up time points. There were no severe complications in this trial, and transient minor adverse effects occurred equally in the three groups. In conclusion, ultrasound-guided PDI with $4 \mathrm{ml}$ of $5 \%$ dextrose provided better efficacy than with 1 and $2 \mathrm{ml}$ based on symptom relief and functional improvement for CTS at the 1st, 4th, and 12th week post-injection, with no reports of severe adverse effects. There was no significant difference between the three groups at the 24th-week post-injection follow-up.

Clinical Trial Registration: www.ClinicalTrials.gov, identifier NCT03598322. 


\section{INTRODUCTION}

Carpal tunnel syndrome (CTS), the most common compressive mononeuropathy caused by entrapment of the median nerve, leads to functional impairment of the hand (Atroshi et al., 1999). Possible etiologies include increased pressure in the intracarpal canal, which compromise the circulation of median nerve (Bland, 2005) and tissue adhesion around median nerve (LaBan et al., 1986; Smith et al., 2008). As for treatment, wrist resting splint and steroid injection have long been the mainstream of conservative treatments, while surgical intervention was reserved for severe or refractory cases (Huisstede et al., 2014). Recently, however, perineural dextrose injection (PDI) has been reported to be beneficial or even better than steroid injection (Wu et al., 2017b; Wu et al., 2018). To explain the therapeutic response of PDI with the hypoosmolar 5\% dextrose for neuropathy-related pain, sensorineural mechanism was postulated that analgesic effect of dextrose on tender peripheral nerves as well as central nerve system via caudal epidural injection (ManiquisSmigel et al., 2016). Potential down-regulation to molecular pathway on the ion channel capsaicin receptor of sensory neurons may attenuate nociceptive and neuropathic pain (Watabiki et al., 2011; Bertrand et al., 2015).

PDI pertains to nerve mobilization, a broader proposition for CTS treatment, which facilitates adhesion release (Page et al., 2012). Perineural injection to separate the median nerve from the flexor retinaculum and flexor tendons at the carpal tunnel inlet with normal saline was reported beneficial to CTS (Wu et al., 2019). Nerve mobilization or hydrodissection implies that adhesiolysis of the median nerve from surrounding tissues may play a role in treating CTS. In clinical practice, we also observed better detachment of the median nerve from surrounding tissues in the carpal tunnel with a larger injecting volume.

However, previous studies mostly focused on injectate content (steroid, 5\% dextrose water, platelet-rich plasma, etc.), where the injecting volumes vary between studies (from 1 to $5 \mathrm{ml}$ ) (Armstrong et al., 2004; Peters-Veluthamaningal et al., 2010; Atroshi et al., 2013; Wu et al., 2017a; Wu et al., 2018). In other words, the effect of injecting volume itself was not investigated, and the optimal amount has yet to be determined. In this randomized, double-blinded, three-arm trial, we aimed to investigate whether PDI with different injectate volumes has different effects for CTS participants.

\section{METHODS}

\section{Study Design}

This study was a prospective, parallel three-arm, double-blinded randomized control trial, approved by IRB of our Hospital. The study was conducted in accordance with the Declaration of Helsinki. The enrollment started in June 2018, and all follow-ups were completed by December 2019. All participants provided written informed consent. The participants and the outcome assessor were blinded for treatment allocation, while the physiatrist performing the injection was the only one aware of the treatment allocations.

\section{Sample Size}

A preliminary power analysis using G-power 3.1.9.4 (University of California, Los Angeles) was calculated in an analysis of variance for comparison of three groups. For an effect size of $0.41(1-\beta)=0.8$ and $\alpha=0.05$, data for at least 60 wrists were required to achieve sufficient power.

\section{Inclusion and Exclusion Criteria}

Inclusion criteria were 1) aged 20-80 years and 2) diagnosed with idiopathic CTS. The participants were required to fulfill the electrophysiological criteria and at least one of the symptoms and signs which are to be described. Abnormal electrophysiological analysis was defined as at least one of the following criteria: 1) a distal motor latency of the median nerve of more than $3.6 \mathrm{~ms}$ at a distance of approximately $7 \mathrm{~cm}$ from the abductor pollicis brevis muscle with median nerve stimulation at the wrist; 2) a sensory nerve conduction velocity (SNCV) from digit-to-wrist segment $(14 \mathrm{~cm})$ of less than $40 \mathrm{~m} / \mathrm{s}$ or an SNCV from mid-palm-to-wrist segment $(8 \mathrm{~cm})$ of less than $37 \mathrm{~m} / \mathrm{s}$. Symptoms and signs included 1) pain or paresthesia in the median nerve innervated area (at least two digits with such symptoms between the thumb and the 4 th digit) for more than 2 months; 2) positive Phalen test, Tinel sign, or flick sign (Chang et al., 2008; Atroshi et al., 2013). Exclusion criteria were previous wrist surgery, traumatic wrist injury within 2 years, previous wrist injection within 3 months, history of peripheral nerve injuries (brachial plexopathy, cervical radiculopathy or thoracic outlet syndrome), history of thyroid or autoimmune disease, and inability to cooperate with study protocol.

\section{Randomization}

Among the 43 participants with 67 wrists that were assessed for eligibility into the trial, four participants were excluded, as they did not meet the inclusion criteria. Therefore, a total of 63 wrists were randomized into three groups, each with 21 wrists (Figure 1). We performed randomization in permuted blocks of six, and the independent research assistant, who was not involved in the eligibility selection process of participants, prepared and sealed the covered envelopes containing the intervention allocation. The participants were assigned to either 1-ml group, 2-ml group or 4-ml group and all of them received a corresponding session of ultrasound-guided PDI. After the injection, all participants were allowed to take simple analgesics (paracetamol), but non-steroidal anti-inflammatory drugs or neuropathic pain medications were prohibited. We didn't provide any other types of therapy or suggestion including physiotherapy, occupational therapy or night splint.

Participants were allowed to receive a second injection by the same physiatrist within a 6-months follow-up period, which was considered as recurrence.

\section{Ultrasound-Guided PDI}

All ultrasound-guided PDIs were performed by a physiatrist with experience of more than 8,000 cases of ultrasound-guided intervention, using Toshiba Aplio 500 platinum ultrasound system. All participants were positioned with the palm facing upward, and median nerve was identified at the level of proximal 


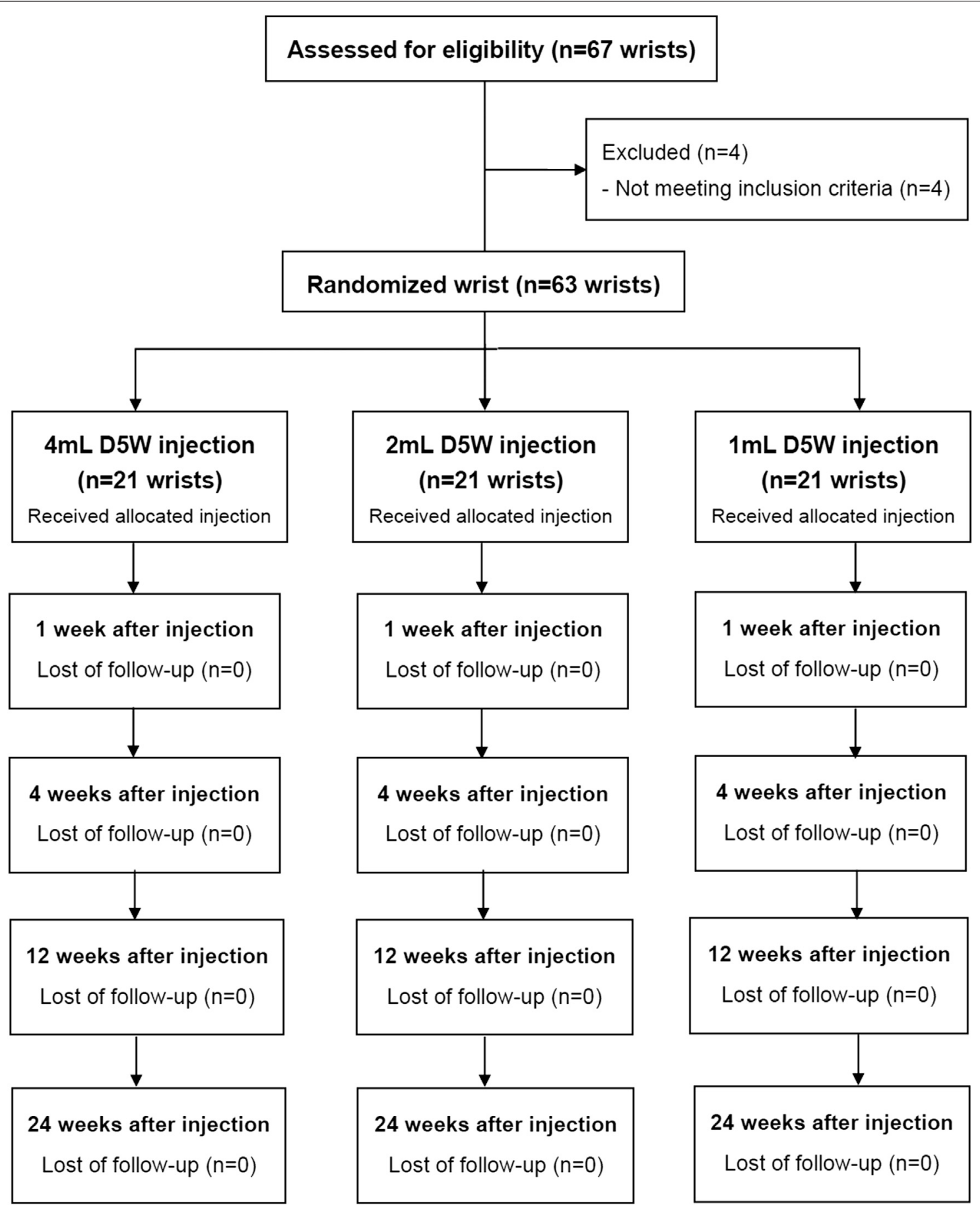

FIGURE 1 | Study flow diagram. D5W, 5\% dextrose water.

inlet (between the pisiform and scaphoid bones). The ultrasoundguided injection was performed using the radial in-plane approach with a 25-gauge needle. After placing the needle between the median nerve and flexor retinaculum, half of the $5 \%$ dextrose water (D5W) was injected to separate the two. The other half of the D5W was then applied below and around the median nerve to ensure that it was surrounded by $\mathrm{D} 5 \mathrm{~W}$ and separated from other tendons within the carpal tunnel. The total D5W injected was either 1, 2 or $4 \mathrm{ml}$. The syringe was covered with white paper and the participants were asked to close their eyes so they remained unaware of the injection amount. All participants were observed for $10 \mathrm{~min}$ after injection for possible immediate complications such as bleeding or worsening paresthesia (Wu et al., 2017a). 


\section{Outcome Assessment}

Baseline assessment includes demographics, medical history, symptom duration, lesion side and result of Phalen and Tinel tests. With the outcome assessor blinded to allocation, all participants were assessed with visual analog scale (VAS), Boston Carpal Tunnel Questionnaire (BCTQ) symptom severity scale and functional status scale; the 11-item QuickDASH (disabilities of the arm, shoulder and hand questionnaire), cross-sectional area (CSA) of the median nerve by ultrasound and electrophysiological evaluation at postinjection at the 1 st, 4 th, 12 th, and 24 th week.

\section{Primary Outcome: VAS}

The continuous VAS scale was used to evaluate pain or paresthesia severity as primary outcome. A score of 10 means unbearable, and a score of 0 means no pain/paresthesia at all. We adopted the maximal pain VAS as score measurement.

\section{Secondary Outcome: BCTQ and QuickDASH}

BCTQ Score is the most commonly used questionnaire for CTS. It includes two subsets of scale, named "symptom severity scale" and "functional status scale (Levine et al., 1993)." The symptom severity scale includes 11 questions, which measure the severity, duration and frequency of daytime and nighttime symptoms, while the functional status scale includes eight questions, which assess the difficulty in performing activities of daily living (ADL). Each question was answered on a scale of $1-5$, with 1 being no symptoms or functional disability, and 5 being most severe and unable to perform certain activities. While the original article uses the mean of all the questions, we used the sum of all the questions. QuickDASH measures the performance of ADL, upper extremity discomfort severity, and the severity of hindered sleep and social life (Beaton et al., 2005).

\section{Secondary Outcome: CSA of Median Nerve}

Ultrasound measurements of the median nerve CSA may be helpful for diagnosis and treatment effect follow-up in CTS (Tai et al., 2012; Wang et al., 2018). At the carpal tunnel inlet level (between the pisiform and scaphoid bones), the CSA was calculated by ultrasound machine after using a caliper to encompass the median nerve manually.

Secondary Outcome: Electrophysiological Evaluation The distal motor latency of the thenar muscles was measured with median nerve stimulation at the wrist at a $7 \mathrm{~cm}$ distance; and the orthodromic SNCV was also measured. The detailed method of measurement is the same as described above.

\section{Statistical Analyses}

All data analyses were done using IBM SPSS Statistics Version 22. Because a preliminary Shapiro-Wilks test demonstrated that samples followed a normal distribution, we decided to analyze demographic data between groups with one-way ANOVA for continuous data and Chi-square test for categorical data. Repeated measures ANOVA were used for analyzing the followup data compared with the baseline. One-way ANOVA was also used for the comparison between groups during follow-up, for
VAS score, BCTQ (symptom severity and functional status), QuickDASH, CSA of median nerve and electrophysiological studies. All statistical tests were two-tailed, and a $p$ value of less than 0.05 was considered statistically significant.

\section{RESULTS}

\section{Clinical Characteristics}

A total of 63 wrists (21 wrists in each group) were analyzed. All participants received a completed follow-up of up to 24 weeks after injection. No significant difference was observed between the three groups in all variables, including age, gender, hypertension, diabetes, symptom duration, Phalen or Tinel test positive rate, lesion side, VAS, BCTQ, QuickDASH, parameters of electrodiagnosis or CSA (Table 1).

\section{Within-Group PDI Effects}

In every group, we observed VAS, BCTQ and QuickDASH significantly improved from baseline data at all follow-up time-points (Table 2 and Supplementary Table S1). For parameters of electrodiagnosis, however, there was no significant difference compared to baseline except for SNCV (finger-wrist) change in the 4-ml group. The median nerve CSA decreased from baseline significantly in both the 2 and 4ml groups (Supplementary Tables S2,S3).

\section{Between-Group PDI Effects: Primary Outcome}

In the 4-ml group, the mean change of VAS from baseline showed a greater improvement than the other groups at the 4th week [4 ml: -3.5 (SD 4.9), $2 \mathrm{ml}:-1.9$ (SD 6.3), $1 \mathrm{ml}:-1.3$ (SD 2.0)] and 12th week [ $4 \mathrm{ml}:-3.9$ (SD 2.9), $2 \mathrm{ml}:-2.4$ (SD 7.4), $1 \mathrm{ml}:-1.7$ (SD 2.4)] of post-injection follow-up. There was no significant difference between the three groups at the 1st and 24th weeks of post-injection follow-up (Table 3 and Figure 2).

\section{Between-Group PDI Effects: Secondary Outcome}

In the 4-ml group, the mean change of BCTQ from baseline improved more compared to other groups at the 1 st week [ $4 \mathrm{ml}$ : -15.4 (SD 8.8) vs. $2 \mathrm{ml}:-7.8$ (SD 11.2) vs. $1 \mathrm{ml}:-8.2$ (SD 7.1)], 4th week [ $4 \mathrm{ml}:-20.4$ (SD 9.5) vs. $2 \mathrm{ml}:-11.3$ (SD 12.9) vs. $1 \mathrm{ml}:-8.5$ (SD 6.7)] and 12th week [4 ml: -20.5 (SD 9.8) vs. $2 \mathrm{ml}:-12.6$ (SD 14.2 ) vs. $1 \mathrm{ml}:-9.9$ (SD 9.7)] post-injection. There was no significant difference, however, between the three groups at the 24th week post-injection (Table 3 and Figure 2).

For the mean change of QuickDASH from baseline, similar to BCTQ results, the 4-ml group yielded more improvement than other groups at the 1st, 4 th and 12th weeks post-injection, but not at the 24th week (Table 3 and Figure 2).

As for the parameters of electrodiagnosis (distal motor latency and SNCV) and median nerve CSA, there was no significant difference between the three groups at any follow-up time points (Table 3 and Figure 2). 
TABLE 1 | Baseline characteristics between three groups.

\begin{tabular}{|c|c|c|c|c|}
\hline & $4 \mathrm{ml}$ group $(n=21)$ & $2 \mathrm{ml}$ group $(n=21)$ & $1 \mathrm{ml}$ group $(n=21)$ & $p$-Value ${ }^{a}$ \\
\hline Age (SD) & $58.4(9.6)$ & $55.2(10.7)$ & $60.3(8.6)$ & 0.231 \\
\hline Female (\%) & 95.2 & 81.0 & 81.0 & 0.311 \\
\hline Hypertension (\%) & 28.6 & 28.6 & 47.6 & 0.327 \\
\hline $\mathrm{DM}(\%)$ & 19.0 & 19.0 & 28.6 & 0.692 \\
\hline Symptoms duration (SD) & $54.4(72.3)$ & $20.6(28.2)$ & $49.8(60.9)$ & 0.134 \\
\hline Phalen or tinel test positive (\%) & 42.9 & 52.4 & 38.1 & 0.638 \\
\hline Lesion side (left, \%) & 45.0 & 40.0 & 61.9 & 0.349 \\
\hline Primary outcome: VAS & $5.4(1.5)$ & $5.9(2.1)$ & $5.4(1.8)$ & 0.797 \\
\hline \multicolumn{5}{|l|}{ Secondary outcome } \\
\hline BCTQ (SD) & $43.6(10.4)$ & $40.8(15.5)$ & $38.4(14.6)$ & 0.902 \\
\hline BCTQ-S (SD) & $24.9(6.6)$ & $24.1(9.3)$ & $23.7(10.0)$ & 0.881 \\
\hline BCTQ-F (SD) & $18.7(5.5)$ & $16.7(5.8)$ & $14.7(5.2)$ & 0.134 \\
\hline QuickDASH (SD) & $24.3(6.8)$ & $23.1(9.9)$ & $22.0(8.3)$ & 0.666 \\
\hline \multicolumn{5}{|l|}{ Electrodiagnosis (SD) } \\
\hline Motor DL, ms & $5.6(1.6)$ & $5.5(1.5)$ & $5.4(1.6)$ & 0.925 \\
\hline SNCV Finger-wrist & $30.2(7.2)$ & $31.5(7.9)$ & $32.7(6.6)$ & 0.432 \\
\hline SNCV Palm-wrist & $25.1(4.7)$ & $26.9(5.8)$ & $26.4(7.0)$ & 0.572 \\
\hline CSA (SD), $\mathrm{mm}^{2}$ & $14.11(2.60)$ & $14.8(3.8)$ & $15.3(4.4)$ & 0.355 \\
\hline
\end{tabular}

D5W, 5\% dextrose water; SD, standard deviation; DM, diabetes mellitus; VAS, visual analog scale; BCTQ, Boston Carpal Tunnel Syndrome Questionnaire (F, function; S, symptom severity); DL, distal latency of median nerve; SNCV, sensory nerve conduction velocity (finger-wrist, finger to wrist segment; palm-wrist, palm to wrist segment); CSA, cross-sectional area of median nerve.

${ }^{a}$ Between-group comparison: one-way ANOVA for continuous data and chi-square test for categorical data.

\begin{tabular}{|c|c|c|c|c|c|c|}
\hline VAS & Baseline & $1 W$ & $4 W$ & $12 W$ & $24 W$ & $p$-Value ${ }^{a}$ \\
\hline $\begin{array}{l}1 \mathrm{ml} \\
\text { group }\end{array}$ & $5.38(1.83)$ & $3.79(2.37)$ & 4.07 (1.89) & 3.69 (1.87) & $3.48(2.36)$ & $<0.001^{* *}$ \\
\hline $\begin{array}{l}2 \mathrm{ml} \\
\text { group }\end{array}$ & $5.93(2.07)$ & 4.51 (2.39) & $4.00(2.74)$ & $3.50(2.57)$ & $2.79(2.22)$ & $<0.001^{\star \star}$ \\
\hline $\begin{array}{l}4 \mathrm{ml} \\
\text { group }\end{array}$ & $5.40(1.51)$ & $3.02(1.82)$ & $1.88(1.76)$ & 1.55 (1.91) & $2.24(2.34)$ & $<0.001^{\star \star}$ \\
\hline
\end{tabular}

$S D$, standard deviation; DM, diabetes mellitus; VAS, visual analog scale. ${ }^{*} p<0.05,{ }^{* *} p<0.01$

${ }^{a}$ One-way repeated-measures ANOVA was used for within-group analysis. The data was presented as mean (SD).

\section{Recurrence Rate}

The proportion of participants who received a second injection within a 6 -months follow-up period was $23.8 \%$ in 1 -ml group, $23.8 \%$ in $2-\mathrm{ml}$ group, and $28.6 \%$ in $4-\mathrm{ml}$ group. No significant difference was observed among the three groups $(p=0.952)$.

\section{Adverse Effects}

No severe adverse effects, such as infection, persistent bleeding, hematoma or death, were identified in any groups during the follow-up period. No significant difference in the minor symptoms and neuropathic symptoms was observed among the three groups (all $p>0.05$ ) (Table 4).

\section{DISCUSSION}

In this RCT, ultrasound-guided PDI with 1, 2 or $4 \mathrm{ml} \mathrm{D5W}$ could all significantly improve pain and function for CTS. The 4-ml group yielded better VAS reduction at the 4 th and 12 th week and functional enhancement at the 1 st, 4 th, and 12 th week postinjection, compared to the 1 and $2-\mathrm{ml}$ groups. No significant between-group differences were observed in parameters of electrophysiological study or median nerve CSA at any followup time points. There were no severe complications, and transient minor adverse effects occurred similarly in all three groups.

Our study focused on the efficacy of PDI and showed that $4 \mathrm{ml}$ dextrose injection is superior to that of 1 or $2 \mathrm{ml}$ with regard to pain and change in functional outcome. Nevertheless, all three groups benefited from reduced pain and improved function within the 6-months follow-up period despite injection volume. Previous studies showed promising effects of dextrose within 6 months (Wu et al., 2017b) and even better than steroids at 4-6 months post-injection (Wu et al., 2018). The placebo group, however, showed reduced symptoms with saline injection as well; therefore, volume effect should be addressed. To investigate the efficacy of hydrodissection, Bland et al. demonstrated the complicated and multifactorial pathogenesis of CTS comprised external compression to the nerve and internal ischemia/inflammation-induced fibrosis to surrounding soft tissues (Bland and Rudolfer, 2003). Increased subsynovial connective tissue in the carpal tunnel affected nerve compliance and permeability (Ettema et al., 2004), and correlated with clinical symptom severity (Tat et al., 2015). A systematic review documented reduced nerve excursion in CTS people compared to healthy controls (Ellis et al., 2017). One cadaver study showed decreased gliding resistance of the nerve in the carpal tunnel after hydrodissection (Evers et al., 2018). Two clinical RCTs further showed positive effects of hydrodissection on pain, function and CSA (Roghani et al., 2018; Wu et al., 2019). Our study adopted an identical injection drug (5\% dextrose) to eliminate confounding bias of dextrose. We validated that even $1 \mathrm{ml}$ PDI would be effective for CTS, despite $4 \mathrm{ml}$ yielding more 
TABLE 3 | Comparison of mean change from baseline in outcomes between three groups.

\begin{tabular}{|c|c|c|c|c|}
\hline & $4 \mathrm{ml}$ group $(n=21)$ & $2 \mathrm{ml}$ group $(n=21)$ & $1 \mathrm{ml}$ group $(n=21)$ & $p$-Value ${ }^{a}$ \\
\hline \multicolumn{5}{|c|}{ Primary outcome $\triangle$ VAS } \\
\hline 1 week & $-2.38(1.81)$ & $-1.42(1.61)$ & $-1.60(1.63)$ & 0.151 \\
\hline 4 weeks & $-3.52(2.22)$ & $-1.93(2.52)$ & $-1.31(1.41)$ & $0.003^{\star \star}$ \\
\hline 12 weeks & $-3.86(1.69)$ & $-2.43(2.72)$ & $-1.69(1.54)$ & $0.004^{\star \star}$ \\
\hline 24 weeks & $-3.17(2.18)$ & $-3.14(2.37)$ & $-1.90(2.12)$ & 0.119 \\
\hline \multicolumn{5}{|c|}{$\begin{array}{l}\text { Secondary outcome } \\
\qquad \Delta \mathrm{BCTQ}(\mathrm{SD})\end{array}$} \\
\hline 1 week & $-15.43(8.83)$ & $-7.76(11.20)$ & $-8.24(7.09)$ & $0.014^{\star}$ \\
\hline 4 weeks & $-20.38(9.55)$ & $-11.29(12.95)$ & $-8.48(6.67)$ & $0.001^{\star \star}$ \\
\hline 12 weeks & $-20.52(9.81)$ & $-12.62(14.19)$ & $-9.86(9.72)$ & $0.010^{*}$ \\
\hline $\begin{array}{l}24 \text { weeks } \\
\Delta \text { BCTQ-S }\end{array}$ & -15.52 (12.07) & $-14.76(11.93)$ & $-10.62(10.55)$ & 0.340 \\
\hline 1 week & $-8.57(4.88)$ & $-4.95(7.47)$ & $-5.67(4.88)$ & 0.163 \\
\hline 4 weeks & $-12.00(6.90)$ & $-7.52(8.05)$ & $-5.91(5.14)$ & $0.015^{\star}$ \\
\hline 12 weeks & $-12.10(6.16)$ & $-8.62(8.94)$ & $-7.76(7.48)$ & 0.157 \\
\hline $\begin{array}{l}24 \text { weeks } \\
\Delta \text { BCTQ-F }\end{array}$ & $-9.67(8.22)$ & $-9.91(7.23)$ & $-7.95(7.76)$ & 0.674 \\
\hline 1 week & $-6.86(4.14)$ & $-2.81(4.84)$ & $-2.57(2.46)$ & $0.001^{\star \star}$ \\
\hline 4 weeks & $-8.38(4.54)$ & $-3.76(5.64)$ & $-2.57(2.48)$ & $0.0002^{\star *}$ \\
\hline 12 weeks & $-8.43(5.65)$ & $-4.00(6.24)$ & $-2.10(3.32)$ & $0.001^{\star \star}$ \\
\hline $\begin{array}{l}24 \text { weeks } \\
\Delta \text { QuickDA }\end{array}$ & $-5.86(5.10)$ & $-4.86(5.69)$ & $-2.67(3.76)$ & 0.108 \\
\hline 1 week & $-9.33(5.89)$ & $-4.29(6.27)$ & $-4.14(5.49)$ & $0.008^{\star \star}$ \\
\hline 4 weeks & $-11.62(6.70)$ & $-6.48(7.79)$ & $-4.67(5.67)$ & $0.004^{\star \star}$ \\
\hline 12 weeks & $-11.19(6.87)$ & $-6.91(8.73)$ & $-5.19(5.75)$ & $0.027^{\star}$ \\
\hline 24 weeks & $-8.62(6.90)$ & $-8.57(7.08)$ & $-6.81(5.64)$ & 0.599 \\
\hline \multicolumn{5}{|c|}{ Electrodiagnosis (SD) } \\
\hline 1 week & $0.06(0.54)$ & $0.01(0.88)$ & $-0.33(0.96)$ & 0.294 \\
\hline 4 weeks & $0.37(1.09)$ & $-0.13(0.58)$ & $-0.08(0.63)$ & 0.124 \\
\hline 12 weeks & $0.15(0.87)$ & $-0.16(0.62)$ & $-0.08(0.60)$ & 0.375 \\
\hline $\begin{array}{l}24 \text { weeks } \\
\Delta \text { SNCV fin }\end{array}$ & $0.09(0.95)$ & $-0.32(0.72)$ & $-0.09(0.57)$ & 0.263 \\
\hline 1 week & 0.45 (3.66) & $0.80(4.63)$ & $0.00(4.14)$ & 0.841 \\
\hline 4 weeks & 0.55 (3.03) & $1.56(3.43)$ & $-0.48(5.16)$ & 0.307 \\
\hline 12 weeks & $3.11(3.79)$ & 1.59 (3.15) & 0.44 (4.89) & 0.138 \\
\hline $\begin{array}{l}24 \text { weeks } \\
\Delta \text { SNCV pa }\end{array}$ & $3.52(3.71)$ & $2.45(3.79)$ & $0.40(4.32)$ & 0.055 \\
\hline 1 week & 0.69 (3.20) & 0.03 (3.53) & $0.27(2.95)$ & 0.816 \\
\hline 4 weeks & $0.00(3.54)$ & 0.02 (3.88) & 0.26 (3.89) & 0.974 \\
\hline 12 weeks & $1.14(2.80)$ & $-0.54(2.72)$ & $0.25(4.77)$ & 0.370 \\
\hline $\begin{array}{l}24 \text { weeks } \\
\Delta \text { CSA (SD) }\end{array}$ & $1.00(4.11)$ & $0.499(3.18)$ & $0.12(5.28)$ & 0.767 \\
\hline 1 week & $-0.61(2.68)$ & $-0.71(2.19)$ & $-0.42(1.50)$ & 0.917 \\
\hline 4 weeks & $-0.44(3.31)$ & $-0.53(3.04)$ & $-0.53(3.27)$ & 0.996 \\
\hline 12 weeks & $-0.88(2.45)$ & $-1.24(3.15)$ & $-1.37(3.13)$ & 0.879 \\
\hline 24 weeks & $-1.89(2.28)$ & $-2.34(2.96)$ & $-1.63(2.87)$ & 0.720 \\
\hline
\end{tabular}

D5W, 5\% dextrose water; SD, standard deviation; DM, diabetes mellitus; VAS, visual analog scale; BCTQ, Boston Carpal Tunnel Syndrome Questionnaire (F, function; S, symptom severity); DL, distal latency of median nerve; SNCV, sensory nerve conduction velocity (finger-wrist, finger to wrist segment; palm-wrist, palm to wrist segment); CSA, cross-sectional area of median nerve.

${ }^{*} p<0.05,{ }^{* *} p<0.01$.

${ }^{a}$ Between-group comparison: one-way ANOVA was used for statistical analysis.

improvement. The results were compatible with our experience during injection where we observed further expansion of the perineural space and longitudinal drug spreading. Therefore, we believe that volume effect did matter for PDI.

In this study, three groups presented improved pain and function comparing to baseline; nevertheless, only the 1 and 2-ml groups exhibited trends of decreased VAS, BCTQ and QuickDASH up to the 6th month of the follow-up period instead of the 4-ml group (Figure 2). Although the effect of dextrose, conducted by a previous
RCT, lasted for up to 6 months (Wu et al., 2017b), the effect of perineural saline injection showed an improved BCTQ only until the 3rd month post-injection which deteriorated by the 6th month of follow-up (Wu et al., 2019). Similarly, another RCT reported the improvement of BCTQ did not last until the 6th month after hydrodissection (with either steroid or hyaluronidase) (Alsaeid, 2019). In this study, via post-hoc Bonferroni analysis, pain and functional outcomes were not significant between the 3rd and 6th months follow-up in the 1,2 and 4-ml groups (VAS: $p=1.000,0.895$, 


\section{A VAS change from baseline}

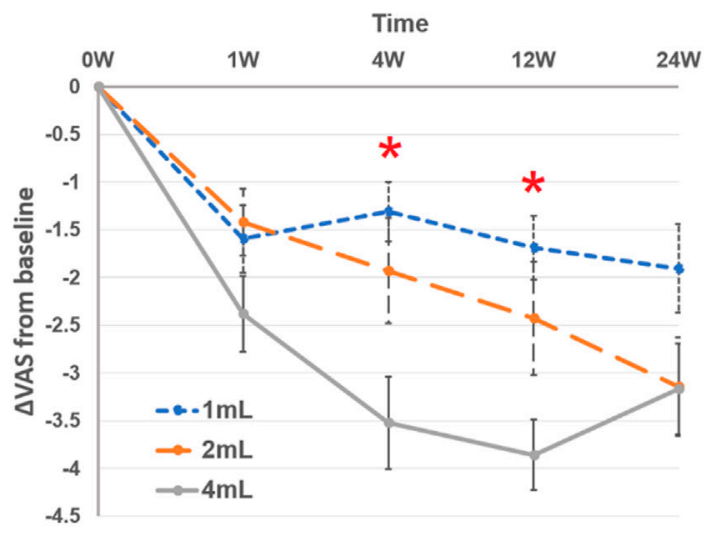

C QuickDASH change from baseline

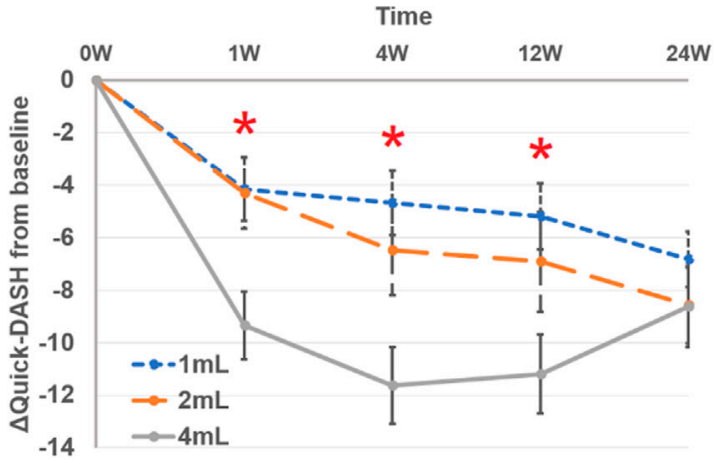

B BCTQ change from baseline

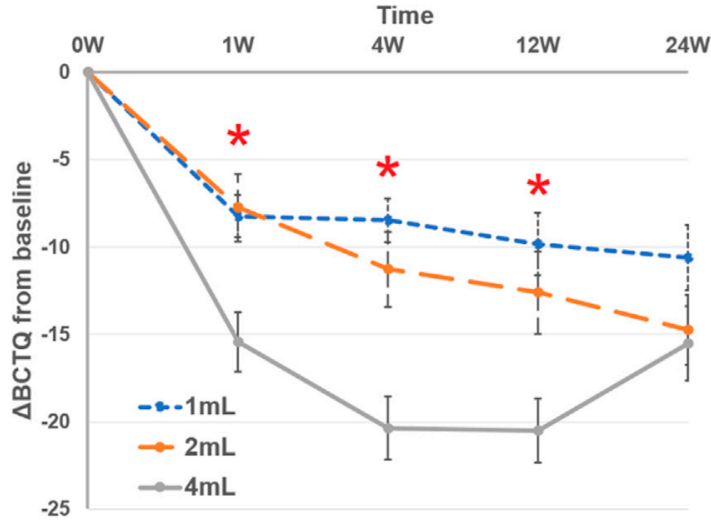

D CSA change from baseline

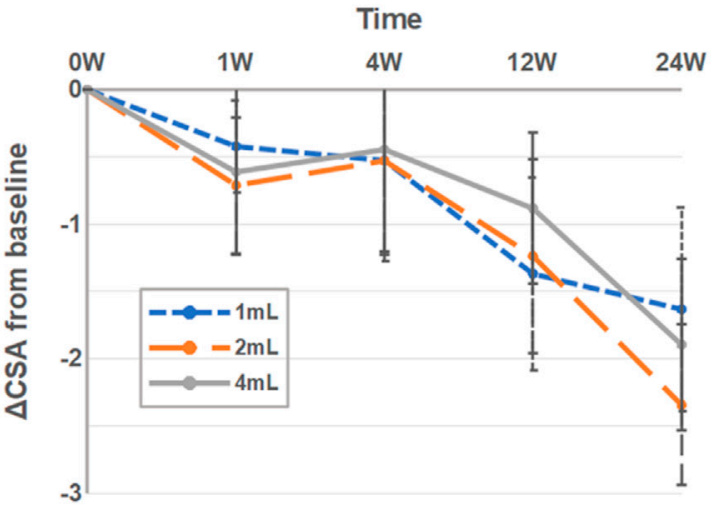

FIGURE 2 | Mean change from baseline between three groups at follow-up time points (1st, 4th, 12th and 24th week) in (A) visual analog scale (VAS), (B) Boston Carpal Tunnel Syndrome Questionnaire (BCTQ) scores, (C) QuickDASH, and (D) cross-sectional area of median nerve (CSA). Asterisk represented significant difference between three groups.

TABLE 4 | Adverse effect.

$4 \mathrm{ml}$ group $2 \mathrm{ml}$ group $1 \mathrm{ml}$ group $p$-Value ${ }^{\mathrm{a}}$

Transient, \%

$\begin{array}{lllll}\text { Minor symptoms }^{\mathrm{b}} & 45.0 & 20.0 & 23.8 & 0.180\end{array}$

$\begin{array}{lllll}\text { Neuropathic symptoms }^{c} & 20.0 & 20.0 & 9.5 & 0.589\end{array}$

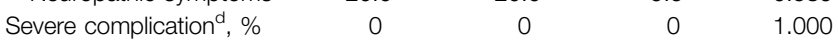

D5W, 5\% dextrose water.

${ }^{a}$ Between-group comparison: chi-square test for categorical data analysis.

${ }^{b}$ Minor symptoms included needling pain, subjective swelling or dizziness subsided

within $10 \mathrm{~min}$.

${ }^{c}$ Neuropathic symptoms included electric shock sensation or finger numbness subsided

within $10 \mathrm{~min}$.

${ }^{d}$ Severe complication included infection, persisted bleeding, hematoma or death.

0.750, respectively; BCTQ: $p=1.000,1.000,0.295$, respectively). Further researches with longer follow-up are necessary to investigate whether the effect of dextrose hydrodissection persists.

We observed significantly reduced CSA of the median nerve by the 6-months follow-up in the 2 and 4-ml groups, instead of improvement in electrophysiological parameters. Many studies investigated different injectates (including steroid, dextrose and platelet-rich plasma) to decrease CSA after injection, even with placebo saline (Wu et al., 2017b; Wang et al., 2018; Shen et al., 2019; Wu et al., 2019). Although not significant, the 1-ml group of our study showed the CSA exhibited a trend of reduction of intraneural inflammation, edema, or swelling of subsynovial connective tissue, which aligns with a previous hypothesis posed by Wang et al. (2018). With the randomization and use of identical injectate, we could infer the volume of hydrodissection mattered.

Although the participants of the 4-ml group complained of minor symptoms, there were no severe adverse effects in any groups. Old studies documented median nerve injury and tendon ruptures after multiple injections (Gottlieb and Riskin, 1980; Kasten and Louis, 1996); however, contemporary ultrasound-guided techniques escalated the accuracy of injection, and were shown to be more effective than blind injection (Lee et al., 2014; Chen et al., 2015). No severe complications were noted in the studies who used the aforementioned ultrasound-guided median nerve injection. While the 4-ml injection delivered more volume of dextrose into the carpal tunnel, potentially resulting in transient higher pressure that may explain the minor symptoms, no participants in the 4-ml group asked for termination of injection. 
Although recurrence rate in 4-ml group was slightly higher than other two groups, no significant difference was observed among the three groups within a 6-months follow-up period. Recent research revealed multiple PDI with high volume dextrose up to $10 \mathrm{ml}$ exerted longer cumulative effects, comparing to their previous study with $5 \mathrm{ml}$ single PDI ( $\mathrm{Li}$ et al., 2020). In our study, we hypothesize the longer symptom duration and motor distal latency of electrodiagnosis in 4-ml group might cause slightly higher recurrence rate than other groups [symptom duration (months): 54.4 in 4-ml group, 20.6 in 2$\mathrm{ml}$ group and 49.8 in 1-ml group; motor distal latency (ms): 5.6 in 4-

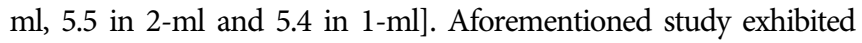
similar PDI results that severe CTS patients had less excellent outcome than mild to moderate CTS patients (Li et al., 2020).

There were several limitations to this study. First, we did not exclude severe CTS patients (Chang et al., 2008). Though we believed randomization would minimize potential bias (19.0, 14.3, 14.3\% in 1, 2 and 4-ml groups, respectively; $p=0.918$ ), the severity of CTS may have obscured the effect of injection. Second, we enrolled participants with bilateral hands, where one hand might influence the functional outcomes of the other hand. Third, this study did not provide direct evidence that larger volume reduced more adhesion around the median nerve. Further studies on the median nerve excursion after PDI are warranted.

\section{CONCLUSION}

Ultrasound-guided PDI with $4 \mathrm{ml}$ D5W provided better efficacy in symptom relief and functional improvement for CTS than with 1 and $2 \mathrm{ml}$ at the 1st, 4 th, and 12th week post-injection, with no reports of severe adverse effects. There was no significant difference between the three groups at the 24th-week post-injection follow-up.

\section{DATA AVAILABILITY STATEMENT}

The raw data supporting the conclusions of this article will be made available by the authors, without undue reservation.

\section{REFERENCES}

Alsaeid, M. A. (2019). Dexamethasone versus hyaluronidase as an adjuvant to local anesthetics in the ultrasound-guided hydrodissection of the median nerve for the treatment of carpal tunnel syndrome patients. Anesth. Essays. Res. 13 (3), 417. doi:10.4103/aer.AER_104_19

Armstrong, T., Devor, W., Borschel, L., and Contreras, R. (2004). Intracarpal steroid injection is safe and effective for short-term management of carpal tunnel syndrome. Muscle Nerve 29 (1), 82-88. doi:10.1002/mus. 10512

Atroshi, I., Flondell, M., Hofer, M., and Ranstam, J. (2013). Methylprednisolone injections for the carpal tunnel syndrome: a randomized, placebo-controlled trial. Ann. Intern. Med. 159 (5), 309-317. doi:10.7326/0003-4819-159-5201309030-00004

Atroshi, I., Gummesson, C., Johnsson, R., Ornstein, E., Ranstam, J., and Rosén, I. (1999). Prevalence of carpal tunnel syndrome in a general population. JAMA 282 (2), 153-158. doi:10.1001/jama.282.2.153

Beaton, D. E., Wright, J. G., and Katz, J. N., and Upper Extremity Collaborative Group (2005). Development of the QuickDASH: comparison of three item-

\section{ETHICS STATEMENT}

The studies involving human participants were reviewed and approved by National Taiwan University Hospital, Taiwan. The patients/participants provided their written informed consent to participate in this study.

\section{AUTHOR CONTRIBUTIONS}

M-TL, C-LL, and C-HW contributed to research design and conception. M-TL and C-LL analyzed data. $\mathrm{M}-\mathrm{YH}, \mathrm{H}-\mathrm{WH}$, and C-CC helped interpreting the results. M-TL drafted the first edition of the manuscript. All authors revised, and agreed the manuscript.

\section{FUNDING}

The research was funded by National Taiwan University Hospital (NTUH 108-M4366, 109-M4670) and Ministry of Science and Technology, Taiwan (MOST 107-2314-B-002-045-MY3, MOST 107-2314-B-002-232-MY3).

\section{ACKNOWLEDGMENTS}

The authors acknowledge statistical assistance provided by the Center of Statistical Consultation and Research in the Department of Medical Research, National Taiwan University Hospital.

\section{SUPPLEMENTARY MATERIAL}

The Supplementary Material for this article can be found online at: https://www.frontiersin.org/articles/10.3389/fphar.2020.625830/ full\#supplementary-material.

reduction approaches. JBJS 87 (5), 1038-1046. doi:10.2106/00004623200505000-00015

Bertrand, H., Kyriazis, M., Reeves, K. D., Lyftogt, J., and Rabago, D. (2015). Topical mannitol reduces capsaicin-induced pain: results of a pilot-level, double-blind, randomized controlled trial. PM R 7 (11), 1111-1117. doi:10.1016/j.pmrj.2015. 05.002

Bland, J. D. (2005). Carpal tunnel syndrome. Curr. Opin. Neurol. 18 (5), 581-585. doi:10.1097/01.wco.0000173142.58068.5a

Bland, J., and Rudolfer, S. (2003). Clinical surveillance of carpal tunnel syndrome in two areas of the United Kingdom, 1991-2001. J. Neurol. Neurosurg. Psychiatry 74 (12), 1674-1679. doi:10.1136/jnnp.74.12.1674

Chang, C.-W., Wang, Y.-C., and Chang, K.-F. (2008). A practical electrophysiological guide for non-surgical and surgical treatment of carpal tunnel syndrome. J. Hand Surg. Eur. Vol. 33 (1), 32-37. doi:10.1177/ 1753193408087119

Chen, P.-C., Chuang, C.-H., Tu, Y.-K., Bai, C.-H., Chen, C.-F., and Liaw, M.-Y. (2015). A Bayesian network meta-analysis: comparing the clinical effectiveness of local corticosteroid injections using different treatment strategies for carpal tunnel syndrome. BMC Musculoskelet. Disord. 16 (1), 363. doi:10.1186/s12891015-0815-8 
Ellis, R., Blyth, R., Arnold, N., and Miner-Williams, W. (2017). Is there a relationship between impaired median nerve excursion and carpal tunnel syndrome? A systematic review. J. Hand Ther. 30 (1), 3-12. doi:10.1016/j. jht.2016.09.002

Ettema, A. M., Amadio, P. C., Zhao, C., Wold, L. E., and An, K.-N. (2004). A histological and immunohistochemical study of the subsynovial connective tissue in idiopathic carpal tunnel syndrome. JBJS 86 (7), 1458-1466. doi:10. 2106/00004623-200407000-00014

Evers, S., Thoreson, A. R., Smith, J., Zhao, C., Geske, J. R., and Amadio, P. C. (2018). Ultrasound-guided hydrodissection decreases gliding resistance of the median nerve within the carpal tunnel. Muscle Nerve 57 (1), 25-32. doi:10.1002/mus.25723

Gottlieb, N. L., and Riskin, W. G. (1980). Complications of local corticosteroid injections. JAMA 243 (15), 1547-1548. doi:10.1001/jama.1980.03300410035021

Huisstede, B. M., Friden, J., Coert, J. H., and Hoogvliet, P. (2014). Carpal tunnel syndrome: hand surgeons, hand therapists, and physical medicine and rehabilitation physicians agree on a multidisciplinary treatment guidelineresults from the European HANDGUIDE Study. Arch. Phys. Med. Rehabil. 95 (12), 2253-2263. doi:10.1016/j.apmr.2014.06.022

Kasten, S. J., and Louis, D. S. (1996). Carpal tunnel syndrome: a case of median nerve injection injury and a safe and effective method for injecting the carpal tunnel. J. Fam. Pract. 43 (1), 79-83.

LaBan, M. M., Friedman, N. A., and Zemenick, G. A. (1986). “Tethered” median nerve stress test in chronic carpal tunnel syndrome. Arch. Phys. Med. Rehabil. 67 (11), 803-804. doi:10.5555/uri:pii:0003999386901565

Lee, J. Y., Park, Y., Park, K. D., Lee, J. K., and Lim, O. K. (2014). Effectiveness of ultrasound-guided carpal tunnel injection using in-plane ulnar approach: a prospective, randomized, single-blinded study. Medicine 93 (29), e350. doi:10. 1097/MD.0000000000000350

Levine, D. W., Simmons, B. P., Koris, M. J., Daltroy, L. H., Hohl, G. G., Fossel, A. H., et al. (1993). A self-administered questionnaire for the assessment of severity of symptoms and functional status in carpal tunnel syndrome. JBJS 75 (11), 1585-1592. doi:10.2106/00004623-199311000-00002

Li, T. Y., Chen, S. R., Shen, Y. P., Chang, C. Y., Su, Y. C., Chen, L. C., et al. (2020). Long-term outcome after perineural injection with 5\% dextrose for carpal tunnel syndrome: a retrospective follow-up study. Rheumatology (Oxford). keaa361. doi:10.1093/rheumatology/keaa361

Maniquis-Smigel, L., Dean Reeves, K., and Jeffrey Rosen, H. (2016). Short term analgesic effects of $5 \%$ dextrose epidural injections for chronic low back pain: a randomized controlled trial. Anesth. Pain. Med. 7 (1), e42550. doi:10.5812/ aapm. 42550

Page, M. J., O’Connor, D., Pitt, V., and Massy-Westropp, N. (2012). Exercise and mobilisation interventions for carpal tunnel syndrome. Cochrane Database Syst. Rev. CD009899. doi:10.1002/14651858.CD009899

Peters-Veluthamaningal, C., Winters, J. C., Groenier, K. H., and Meyboom-de Jong, B. (2010). Randomised controlled trial of local corticosteroid injections for carpal tunnel syndrome in general practice. BMC Fam. Pract. 11 (1), 54. doi:10.1186/1471-2296-11-54

Roghani, R. S., Holisaz, M. T., Tarkashvand, M., Delbari, A., Gohari, F., Boon, A. J., et al. (2018). Different doses of steroid injection in elderly patients with carpal tunnel syndrome: a triple-blind, randomized, controlled trial. Clin. Interv. Aging 13, 117. doi:10.2147/CIA.S151290
Shen, Y. P., Li, T. Y., Chou, Y. C., Ho, T. Y., Ke, M. J., Chen, L. C., et al. (2019) Comparison of perineural platelet-rich plasma and dextrose injections for moderate carpal tunnel syndrome: a prospective randomized, single-blind, head-to-head comparative trial. J. Tissue Eng. Regen. Med. 13 (11), 2009-2017. doi:10.1002/term. 2950

Smith, J., Wisniewski, S. J., Finnoff, J. T., and Payne, J. M. (2008). Sonographically guided carpal tunnel injections: the ulnar approach. J. Ultrasound. Med. 27 (10), 1485-1490. doi:10.7863/jum.2008.27.10.1485

Tai, T.-W., Wu, C.-Y., Su, F.-C., Chern, T.-C., and Jou, I.-M. (2012). Ultrasonography for diagnosing carpal tunnel syndrome: a meta-analysis of diagnostic test accuracy. Ultrasound Med. Biol. 38 (7), 1121-1128. doi:10.1016/ j.ultrasmedbio.2012.02.026

Tat, J., Wilson, K. E., and Keir, P. J. (2015). Pathological changes in the subsynovial connective tissue increase with self-reported carpal tunnel syndrome symptoms. Clin. Biomech. 30 (4), 360-365. doi:10.1016/j.clinbiomech.2015. 02.015

Wang, J. C., Lin, K. P., Liao, K. K., Chang, Y. C., Wang, K. A., Huang, Y. F., et al. (2018). Sonographic median nerve change after steroid injection for carpal tunnel syndrome. Muscle Nerve 58 (3), 402-406. doi:10.1002/mus.26171

Watabiki, T., Kiso, T., Kuramochi, T., Yonezawa, K., Tsuji, N., Kohara, A., et al. (2011). Amelioration of neuropathic pain by novel transient receptor potential vanilloid 1 antagonist AS1928370 in rats without hyperthermic effect. J. Pharmacol. Exp. Ther. 336 (3), 743-750. doi:10.1124/jpet.110.175570

Wu, Y. T., Chen, S. R., Li, T. Y., Ho, T. Y., Shen, Y. P., Tsai, C. K., et al. (2019). Nerve hydrodissection for carpal tunnel syndrome: a prospective, randomized, double-blind, controlled trial. Muscle Nerve 59 (2), 174-180. doi:10.1002/ mus. 26358

Wu, Y.-T., Ho, T.-Y., Chou, Y.-C., Ke, M.-J., Li, T.-Y., Huang, G.-S., et al. (2017a). Six-month efficacy of platelet-rich plasma for carpal tunnel syndrome: a prospective randomized, single-blind controlled trial. Sci. Rep. 7 (1), 94 doi:10.1038/s41598-017-00224-6

Wu, Y.-T., Ho, T.-Y., Chou, Y.-C., Ke, M.-J., Li, T.-Y., Tsai, C.-K., et al. (2017b). Six-month efficacy of perineural dextrose for carpal tunnel syndrome: a prospective, randomized, double-blind, controlled trial. Mayo Clin. Proc. 92 (8), 1179-1189. doi:10.1016/j.mayocp.2017.05.025

Wu, Y. T., Ke, M. J., Ho, T. Y., Li, T. Y., Shen, Y. P., and Chen, L. C. (2018). Randomized double-blinded clinical trial of 5\% dextrose versus triamcinolone injection for carpal tunnel syndrome patients. Ann. Neurol. 84 (4), 601-610. doi:10.1002/ana.25332

Conflict of Interest: The authors declare that the research was conducted in the absence of any commercial or financial relationships that could be construed as a potential conflict of interest.

Copyright (c) Lin, Liao, Hsiao, Hsueh, Chao and Wu. This is an open-access article distributed under the terms of the Creative Commons Attribution License (CC BY). The use, distribution or reproduction in other forums is permitted, provided the original author(s) and the copyright owner(s) are credited and that the original publication in this journal is cited, in accordance with accepted academic practice. No use, distribution or reproduction is permitted which does not comply with these terms. 